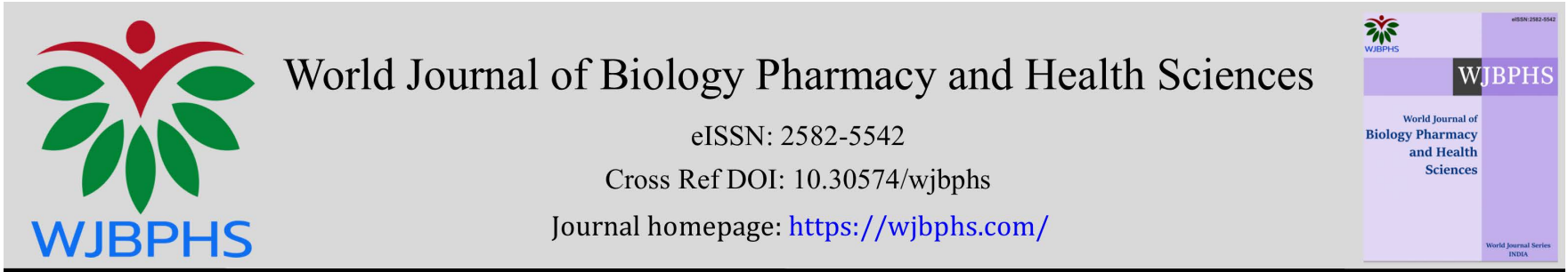

(RESEARCH ARTiCLE)

\title{
Behavioral and biochemical changes in Wister albino rats exposed to different brands of vulcanizer solutions
}

\author{
Zephaniah H Shenia 1, Otitoju Olawale 2, Moses A Abah 2,*, Emochone R Yohanna 2, Emmanuel C Okoli 2 \\ Bilyaminu Habibu ${ }^{2}$ and Asemave S Saaku ${ }^{2}$ \\ ${ }^{1}$ Department of Biochemistry, University of Nigeria Nsukka, Nsukka, Enugu State, Nigeria. \\ 2 Department of Biochemistry, Faculty of Pure and Applied Sciences, Federal University Wukari, Taraba State, Nigeria.
}

World Journal of Biology Pharmacy and Health Sciences, 2022, 09(02), 021-027

Publication history: Received on 02 January 2022; revised on 10 February 2022; accepted on 12 February 2022

Article DOI: https://doi.org/10.30574/wjbphs.2022.9.2.0034

\begin{abstract}
Volatile solvents are liquids that vapourize at room temperature and are found in a multitude of inexpensive, easily available products used for common household and industrial purposes. There is an increased abuse of inhalants among the youths in Nigeria for the purpose of mind-alteration or getting high. Vulcanizer solutions are one of the widely used inhalants that lack proper labelling hence there may be adulteration of these substances which could also be dangerous to health of the sniffers. The objective of this research was to subject wistar albino rats to different brands of vulcanizer solutions to investigate the possible effects these solutions have on social behaviour as well as significant changes in the concentrations of some liver function enzymes. Commercial assay kit from Randox Laboratories Ltd was used for the determination of the enzymes of interest. The study found that only alanine aminotransferase (ALT) was significantly increased $(\mathrm{P}<0.05)$ and changes in behaviours on exposition to miliki diamond (MD) solutions were observed. Serum ALT, AST and ALP levels have been reported to increase than normal in various hepatocellular damages such as necrosis, hepatitis, etc. However, ALT is more specific for detection of hepatotoxicity, therefore its significant increase, when other enzymes do not increase, indicates that vulcanizer solutions (specifically MD) may contribute enormously to certain liver diseases. The changes in behaviours as well as enzyme concentration could be due to various chemicals constituents (such as benzene, toluene, ethyl, etc) in vulcanizer solutions. Therefore, sniffers of vulcanizer solutions may be highly susceptible to various diseases of the liver and other organs, and this may contribute enormously to the prevalence of liver diseases as being recently reported.
\end{abstract}

Keywords: Vulcanizer Solutions; Liver; Enzymes; Wistar albino rats; Inhalants

\section{Introduction}

The use of drugs and volatile substances among the youths in Nigeria has become prevalent and may be contributing greatly to some of the health and social problems being faced in this generation. Most adolescents and adults in the secondary schools and tertiary institutions have been practically engaged in the abuse of various substances (including tobacco, heroin, cocaine, gases, nitrites, etc), which due to long time or chronic use has led to the dependence (addiction) by these youths. There is an increased use of vulcanizer solutions among the youths in Wukari Taraba State and Nigeria as a whole which may results into some health problems in the general populace. Similarly, various brands of these vulcanizer solutions lack proper labelling hence there may be adulteration of these substances which could also be dangerous to health of the sniffers.

\footnotetext{
* Corresponding author: Moses A Abah

Department of Biochemistry, Faculty of Pure and Applied Sciences, Federal University Wukari, Taraba State, Nigeria.

Copyright $(2022$ Author(s) retain the copyright of this article. This article is published under the terms of the Creative Commons Attribution Liscense 4.0.
} 
According to [1], inhalants are volatile substances that produce chemical vapours that can be inhaled to induce a psychoactive or mind-altering effect. Although other abused substances can be inhaled, the term "inhalants" is used to describe a variety of substances whose main common characteristic is that they are rarely, if ever, taken by any route other than inhalation [1]. Inhalants are volatile substances that produce chemical vapours that can be inhaled to induce a psychoactive or. This definition encompasses a broad range of chemicals that may have different pharmacological and toxicological effects and are found in hundreds of different products which as a result make a precise categorization of inhalants somewhat difficult. Volatile solvents are liquids that vaporize at room temperature and are found in a multitude of inexpensive, easily available products used for common household and industrial purposes, which include paint thinners and removers, dry-cleaning fluids, degreasers, gasoline, glues (e.g. vulcanizer solutions), correction fluids and many more.

Vulcanizer solution has been reported to be made up of toluene, ethyl and benzene [2] and can also be considered as an inhalant (particularly glue). Despite the improper labelling of most vulcanizer solutions, some youths which are usually recognised as drug addicts, alcohol abusers or smokers have found the inhalation of these unknown substances necessary for the purpose of inducing psychoactive or mind-altering effects. The inhalation of these solutions alongside other volatile substances has resulted in an observable changes in the social behaviours of the youths in the community. They can cause serious damages to the integrity of the central nervous system and the neurobiological development [3] hence, the abuse of these substances is one of the most dangerous forms of drug abuse, more pernicious, the most widespread and most toxic which can lead to serious accidents [4]. The abuse of volatile substances and its consequent health problems have been recently reported in Egypt, Israel, Slovakia, Togo and USA [5-7]. In some parts of Nigeria such as Wukari Taraba State, the use of vulcanizer solutions have also been observed among a few number of the youths, which by all indications can be believed to be in practice in other regions around the country. The glues sniffed include diamond rubber solution, temple rubber solution etc, which are produced in Taiwan and used by vulcanizers to paste cars' inner tubes tires when punctured. The Fulani herdsmen have been observed to be the addicted inhalants of vulcanizer solutions which according to most believes by people may have contributed enormously to their presumptuous or overbearing attacks across the nation. The prevalence of these substances could be partly due to easy accessibility, because much money is not required for their purchase [2]. The routes of sniffing can be through the nose or mouth. Vulcanizer solutions produced effects such as slurred speech, inability to coordinate movements, euphoria (intense happiness), dizziness, light headedness, hallucinations, and other states of being misled.

Despite all the health problems reported to be associated with inhalants abuse, a little or no report have been made concerning vulcanizer solutions and their toxicological and/or pharmacological effects. Therefore, it is important to consider the biochemical effects of these solutions in the liver, because the liver being the organ saddled with the responsibility of detoxification of chemicals and metabolism of drugs can be damaged or has it functions impaired as a result of exposure to vulcanizer solutions which may consequently lead to many implications on one's health [8]. These damages are associated with cellular necrosis, increase in tissue lipid peroxidation and depletion of reduced glutathione levels [9]. If the solutions can cause damages to the liver, some of the enzymes aspartate aminotransferase (AST), alanine aminotransferase (ALT), alkaline phosphatase (ALP) etc functioning in the biochemical activities of the liver may be released into the bloodstreams thereby leading to increase in their (the enzymes) concentrations in the blood than normal. Impaired liver and other organs can also lead to increase or decrease in the concentrations of certain biomarkers. [10] Reported that increase in Glutathione-S-transferases (GSTs) level in exposed individuals may indicate a form of damage to one or more organs.

The chronic use of inhalants can be associated with a number of serious health problems ranging from intoxication to even death. Many of the addicted smokers and drinkers also use one or more inhalants and these have posed a number of serious social and health problems on these individuals and the society at large.

\section{Material and methods}

The four different brands of the vulcanizer solutions (miliki diamond, temple, Dr Bond and OSHN diamond) used for this research were bought from new market Wukari, Taraba State.

\subsection{Experimental animals}

A total of twenty (20) healthy male wistar albino rats, weighing 160.4 to $318.2 \mathrm{~g}$ were obtained from the animal house unit of the Department of Biochemistry, Federal University Wukari, and Nigeria. They were kept in a clean cage housed in a well-ventilated room at temperature $25-27^{\circ} \mathrm{C}$ under natural light and dark cycle with free access to food and water. 


\subsection{Experimental design}

Twenty (20) Wister albino rats were randomly divided into five groups (A - E), consisting of four rats each. Each group except $\mathrm{E}$ was allowed to sniff one (1) tube (approximately $10 \mathrm{ml}$ ) of the different brands of samples for 5 minutes and they were fed with water and feed ad libitum. The group E animals (control group) were fed with only normal feed and water. Two animals each from each group were sacrificed after 24hours of exposure (acute toxicity) and blood samples collected into sample bottles for biochemical analysis. The remaining animals were exposed to repeated doses of vulcanizer solutions for additional 14days (chronic toxicity) before sacrifice. The animals were then observed for behavioural changes such as aggressiveness, rise and roughness of fur and fore limbs decrease and increase.

\subsection{Biochemical analysis}

The liver function tests were determined by the colorimetric method of [11] using a commercial assay kit from Randox Laboratories Ltd.

\subsection{Determination of aspartate aminotransferase (AST) activity}

AST was measured by monitoring the concentration of oxaloacetate hydrazone formed with 2, 4-dinitrophenyl hydrazine. The colour intensity was measured against the blank at $546 \mathrm{~nm}$. The blank and sample test tubes were set up in duplicates. A volume of $0.1 \mathrm{ml}$ of serum was pipette into the sample tubes. $0.5 \mathrm{ml}$ of reagent 1 was pipette into both sample and blank tubes. The solutions were thoroughly mixed and incubated for exactly 30 minutes at $37^{\circ} \mathrm{C}$ and $\mathrm{pH}$ 7.4. $0.5 \mathrm{ml}$ of Reagent 2 containing 2, 4-dinitrophenyl hydrazine was added into all the test tubes followed by $0.1 \mathrm{ml}$ of sample into the blank tubes. $5 \mathrm{ml}$ of $\mathrm{NaOH}$ was then added to each test tube after incubation. The absorbance was read against the blank after 5 minutes at $546 \mathrm{~nm}$.

\subsection{Calculation}

The activity of AST was read from standard curve.

\subsection{Determination of serum alanine aminotransferase (ALT) activity}

ALT was measured by monitoring the concentration of pyruvate hydrazone formed with 2, 4-dinitrophenlyhydrazine. The colour intensity is measured against the blank at $540 \mathrm{~nm}$. The blank and sample test tubes were set up in duplicates. A volume of $0.1 \mathrm{~mL}$ of serum was pipette into the sample tubes. To these were added $0.5 \mathrm{ml}$ buffer solution containing phosphate buffer, L-alanine and $\alpha$-Oxoglutarate. The mixtures were thoroughly mixed and incubated for exactly 30 minutes at $37^{\circ} \mathrm{C}$ and $\mathrm{pH} 7.4$. A volume, $0.5 \mathrm{ml}$ of reagent containing 2, 4-dinitrophenlyhydrazine was later added to both tubes while $0.1 \mathrm{ml}$ of sample was added to the sample blank tube. $5 \mathrm{~mL}$ of $\mathrm{NaOH}$ solution was added to each tube and mixed after 20 minutes of incubation. The absorbance was read against the blank after 5minutes at $540 \mathrm{~nm}$.

\subsection{Calculation}

The activity of ALT was read up from standard curve

\subsection{Determination of serum alkaline phosphatase (ALP) activity}

The blank and sample test tubes were set up in duplicates and $0.05 \mathrm{ml}$ of sample was pipette into a sample test tubes. $0.05 \mathrm{ml}$ of distilled water was pipette into the blank tube. $3.0 \mathrm{ml}$ of the substrate was pipette into each tube respectively, which was then mixed and the initial absorbance taken at $450 \mathrm{~nm}$. The stop watch was started and the absorbance of the sample and the blank read again three more times at one minute intervals.

\subsection{Calculation}

The activity of alkaline phosphatase in the serum was obtained from the formula below:

Activity of ALP (in IU/L) $=\frac{\text { Absorbance of Samples }}{\text { Absorbance of Standard }} \mathrm{X}$ concentration of standard.

\subsection{Statistical analysis}

The results were analyzed using one way analysis of variance (ANOVA) followed by Post-hoc tests. Multiple comparisons using LSD was also performed ( $\mathrm{P} \leq 0.05$ was regarded as significant). The Statistical Package for Social Sciences (SPSS) Computer software version 21was used for the data analysis. 


\section{Results}

\subsection{Result of behavioural changes}

After the acute and chronic exposures, the behaviours of the animals were observed. Immediately after inhalation, the animals were quiet for some time, there was rise of the furs and the animals were aggressive at first but later became weak. The ability of the animals to resist these effects was low. These effects were observed in all the experimental groups except the control group where there were no behavioural effects. The fore limbs and the heads were dull and weak after long time expositions. There was an aggression of the back limbs which later became dull. All the rats were restless and shivering for long period of time. These behavioural changes are similar to those observed in sniffers.

\subsection{Liver function test results under acute exposure}

The result for AST, ALT and ALP is shown in Table 1. The control group (CO) shows highest level of AST (201.5 \pm 12.50 ), followed by ALT (133.5 \pm 76.37$)$, and then ALP (67.35 \pm 12.45$)$. On exposure to miliki diamond (MD), AST is higher $(207.0 \pm 45.00)$ followed by ALT (67.27 \pm 28.50$)$, with ALP being the least (67.27 \pm 18.11$)$. Dr Bond (DB) has highest level of AST (172.5 \pm 33.50$)$, followed by ALT (82.50 \pm 6.500$)$ and the ALP (40.58 \pm 11.01$)$. Similarly, AST was higher $(195.0 \pm 23.00)$ than ALT $(145.0 \pm 7.000)$ and ALP $(47.89 \pm 15.34)$ on exposure to Temple (TP).When exposed to OSHN diamond (OD), AST still was higher in concentration (97.50 \pm 73.50$)$ than ALT (80.00 \pm 7.000$)$ and ALP (71.90 \pm 4.505$)$. In all the samples including the control, AST was shown to be higher than ALT and ALP (Figure 1).

Table 1 Liver function test in wistar albino rats exposed to acute dose of vulcanizer solutions

\begin{tabular}{|c|c|c|c|c|c|c|c|c|c|c|c|c|c|c|c|c|c|c|c|c|c|c|}
\hline \multirow[t]{2}{*}{ G } & \multirow[t]{2}{*}{$\mathbf{R}$} & \multirow[t]{2}{*}{$\mathbf{0}$} & \multirow[t]{2}{*}{$\mathbf{U}$} & \multirow[t]{2}{*}{$\mathbf{P}$} & \multirow[t]{2}{*}{$\mathbf{S}$} & \multirow{2}{*}{\multicolumn{2}{|c|}{$\frac{P}{A}$}} & \multirow{2}{*}{$\frac{\mathbf{R}}{\mathrm{S}}$} & \multirow[t]{2}{*}{$\mathbf{A}$} & \multirow{2}{*}{$\frac{\mathbf{M}}{\mathbf{T}}$} & \multirow{2}{*}{$\frac{\mathbf{E}}{\mathbf{A}}$} & \multirow{2}{*}{\multicolumn{2}{|c|}{$\mathbf{T}$}} & \multirow[t]{2}{*}{$\mathbf{E}$} & \multicolumn{2}{|c|}{$\mathbf{R}$} & \multicolumn{2}{|l|}{$\mathbf{S}$} & & \multirow{2}{*}{$\frac{(\mathrm{U}}{\mathrm{A}}$} & \multirow{2}{*}{$\frac{1}{\mathbf{L}}$} & \multirow{2}{*}{$\begin{array}{l}\mathbf{L} \mathbf{J} \\
\mathbf{P}\end{array}$} \\
\hline & & & & & & & & & & & & & & & $\mathbf{L}$ & & & & $\mathbf{T}$ & & & \\
\hline M & & & & & $\mathrm{D}$ & 20 & 7.0 & \pm & 45 & 00 & & 2 & 3 . & 5 & \pm & 2 & 8 & 5 & 0 & 67.27 & \pm & 18.11 \\
\hline D & & & & & B & 17 & 2.5 & \pm & 33 & 50 & 8 & 2 & .5 & 0 & \pm & 6 & . 5 & 0 & 0 & 40.58 & \pm & 11.01 \\
\hline $\mathrm{T}$ & & & & & $\mathrm{P}$ & 19 & 5.0 & \pm & 23 & 00 & & 4 & 5 . & 0 & \pm & 7 & . 0 & 0 & & 47.89 & \pm & 15.34 \\
\hline 0 & & & & & $\mathrm{D}$ & 97 & .50 & \pm & 73 & 50 & 8 & 0 & .0 & 0 & \pm & 7 & .0 & 0 & 0 & 71.90 & \pm & 4.505 \\
\hline $\mathrm{C}$ & & & & & 0 & 20 & 1.5 & \pm & 12 & 50 & 1 & 3 & 3 & 5 & \pm & 7 & 6 & 3 & 7 & 67.35 & \pm & 12.45 \\
\hline
\end{tabular}

Key: $\mathrm{MD}=$ Miliki Diamond; $\mathrm{DB}=$ Dr. Bond; TP = Temple; $\mathrm{OD}=$ OSHN Diamond; and CO = control

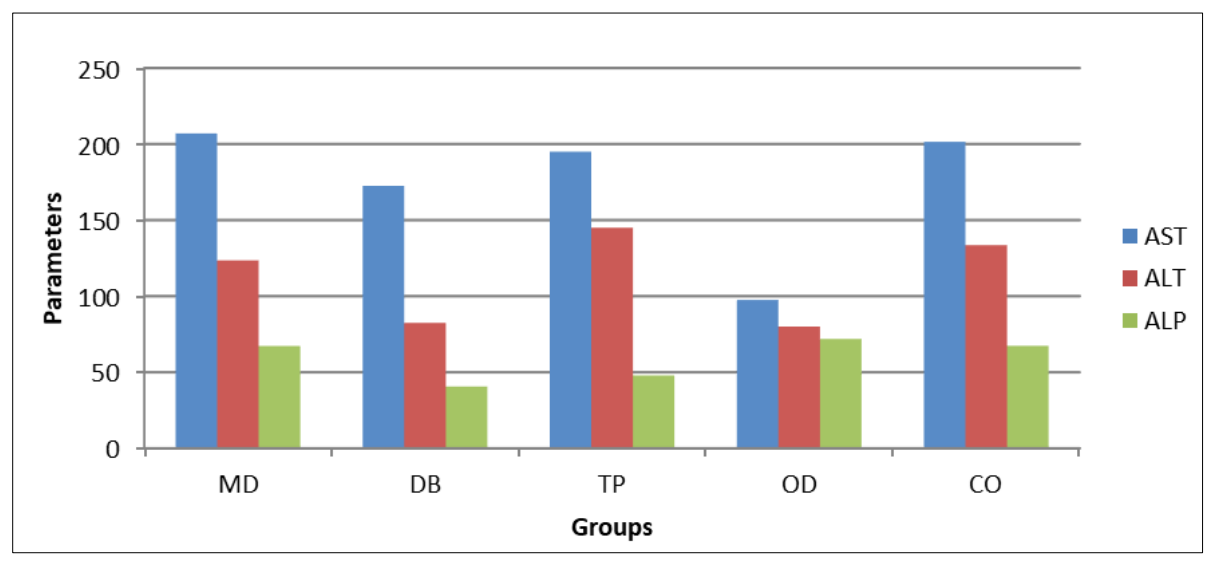

Figure 1 Acute effects of four (4) vulcanizer solutions on liver function enzymes

\subsection{Liver function test results under chronic exposure}

The result of the chronic exposure shows that AST is higher in both the samples and the control. From the result in table 2 , it can be seen that the control group (CO) shows higher level of AST (172.0 \pm 19.65 ) followed by ALT (56.88 \pm 3.359 ) and the ALP (55.30 \pm 11.62$)$. On exposure to miliki diamond (MD), the serum concentration of AST was higher than those of ALT and ALP, with the values of $216.7 \pm 23.93,115.8 \pm 21.86$ and $60.45 \pm 18.67$ respectively. In DB, AST has a 
higher value $(190.1 \pm 13.15)$ followed by ALT $(88.33 \pm 9.600)$ and then ALP $(47.31 \pm 2.524)$. Similarly, AST was also higher than ALT and ALP and exposure to TP and OD (Figure 2).

Table 2 Liver function test in wistar albino rats exposed to chronic dose of vulcanizer solutions

\begin{tabular}{|c|c|c|c|c|c|c|c|c|c|c|c|c|c|c|}
\hline \multirow[t]{2}{*}{$\mathbf{G}$} & \multirow[t]{2}{*}{$\mathbf{R}$} & \multirow[t]{2}{*}{$\mathbf{0}$} & \multirow[t]{2}{*}{$\mathbf{U}$} & \multirow[t]{2}{*}{$\mathbf{P}$} & \multirow[t]{2}{*}{$S$} & A & $\mathbf{R}$ & A & $\mathbf{T}$ & $\mathbf{E}$ & $\mathbf{R}$ & $\mathbf{U}$ & / & $\mathbf{L}$ \\
\hline & & & & & & $\mathbf{A}$ & $\mathbf{S}$ & $\mathbf{T}$ & A & $\mathbf{L}$ & $\mathbf{T}$ & A & $\mathbf{L}$ & $\mathbf{P}$ \\
\hline M & & & & & $\mathrm{D}$ & 216.7 & \pm & 23.93 & 115.8 & \pm & 21.86 & 60.45 & \pm & 18.67 \\
\hline $\mathrm{D}$ & & & & & B & 190.1 & \pm & 13.15 & 88.33 & & 9.600 & 47.31 & \pm & 2.524 \\
\hline $\mathrm{T}$ & & & & & $\mathrm{P}$ & 231.5 & \pm & 44.50 & 86.83 & \pm & 12.00 & 50.91 & \pm & 4.265 \\
\hline 0 & & & & & D & 168.0 & \pm & 16.68 & 91.97 & \pm & 5.825 & 56.00 & \pm & 10.13 \\
\hline $\mathrm{C}$ & & & & & 0 & 172.0 & \pm & 19.65 & 56.88 & \pm & 3.359 & 55.30 & \pm & 11.62 \\
\hline
\end{tabular}

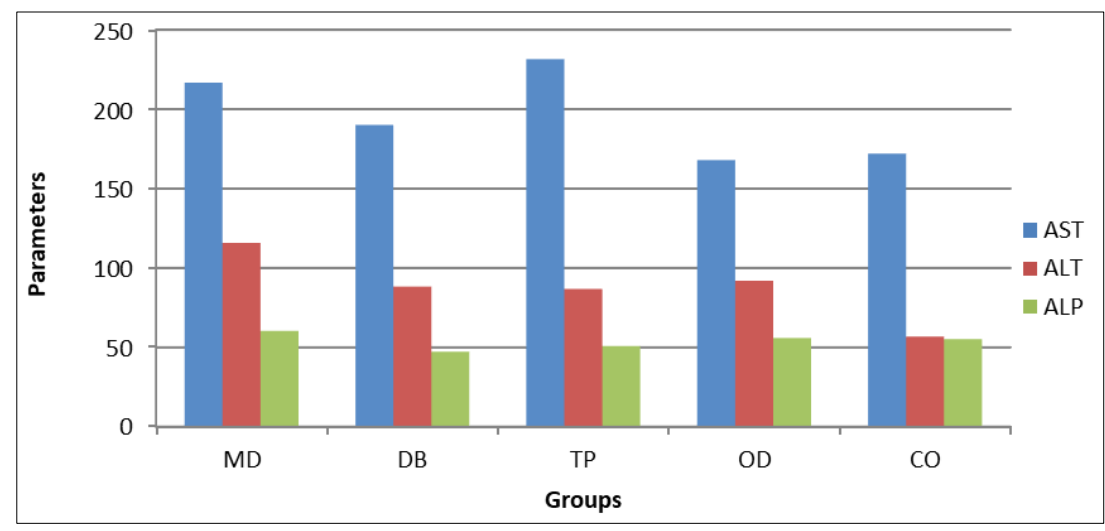

Figure 2 Acute effects of four vulcanizer solutions on liver function enzymes

\section{Discussion}

Volatile substances abuse has been considered as one of the most dangerous forms of drug abuse. Among these substances, vulcanizer solution has received an increased use in Wukari and Nigeria at large, and this has been associated with certain medical and social problems.

The liver plays a key role in the metabolic processes of itself as well as other tissues in maintaining the internal body environment (homeostasis). Hepatic injury due to some toxic substances found in many industrial products and failure to eliminate these metabolic products by the liver often results in marked distortion of the normal function of the liver [12]. Aspartate aminotransferase (AST) and alanine aminotransferase (ALT) in the serum are liver enzymes that participate in many of the activities of the liver and hence have been found to be often associated with hepatocellular damage [13]. Serum alkaline phospatase (ALP) is a sensitive detector in biliary cirrhosis, hepatitis and in diseases characterized by inflammation, regeneration, intrahepatic and extrahepatic bile obstruction.

In this study, both behavioural and medical effects of four different brands of vulcanizer solutions on Wistar albino rats have been shown by evaluating the animals' behaviours and serum concentrations of liver enzymes after short and longterms exposures. The behaviours of the animals were shown to be affected on exposure to these substances. Similar to those reported in the literature $[1,14,15]$, depression including dizziness, disorientation, aggression, weakness or sleeping, etc within few seconds to minutes of inhalation have been observed. Upon long time exposition the observed changes were rise of fur and roughness of fur, dullness and weakness of fore limbs and heads, restlessness and shivering. Despite the behavioural consequences observed and reported, relatively little is known about the mechanisms of the behavioural effects of glue abuse, although drug abuse generally have been reported in the literature to act on brain mechanisms that cause a high-risk individual to engage in aggressive and violent behaviour [16]. After a long period of exposure decreased in the effects were observed and this could be as a result of adaptation or tolerance by the animals. Therefore, people who abuse in the glue-sniffing would risk developing a tolerance, which means that a larger quantity 
would be needed by sniffers in order to have the desired effects. Because of this, sniffers increase their daily doses of glues with prolongs abuse.

The results for acute exposure of all the vulcanizer solutions have shown decrease (except the miliki diamond solution (MD)) in the serum concentration of AST. On ALT, only the temple solution (TP) increases the serum concentration of AST while all other solutions have decreasing effects on this liver enzyme. For ALP, all except OSHN diamond solution (OD) have decreasing effects. Despite the increasing and decreasing effects of these solutions, they have been shown to be statistically not significant $(\mathrm{P}<0.05)$ when compared with those of the control. On long-time exposure only the miliki diamond (MD) has significantly increased on ALT. A similar study also showed a significant increase in the serum levels of ALT, AST and ALP on exposure to diamond glue [2], and hence one can suggest that the MD after long period (14days) had marked adverse effect on the liver of the treated rats. ALT is primarily found in liver and is the most specific and frequently relied biomarker of hepatotoxicity [12], hence, since only its level (among others, AST and ALP) was significantly elevated in this study, it is then suitable to suggest that exposure of wistar albino rats to MD results to liver damages.

\section{Conclusion}

The changes in behaviours as well as enzyme concentration could be due to various chemicals constituents (such as benzene, toluene, ethyl, etc) in vulcanizer solutions. Therefore, sniffers of vulcanizer solutions may be highly susceptible to various diseases of the liver and other organs, and this may contribute enormously to the prevalence of liver diseases as being recently reported.

\section{Compliance with ethical standards}

\section{Acknowledgments}

We want to express our profound gratitude to Professor Otitoju Olawale, the Laboratory head, for his unwavering support and guidance, and all those who contributed directly to this study.

\section{Disclosure of conflict of interest}

The authors state that there was no conflict of interest.

\section{Statement of ethical approval}

Ethical approval was obtained from ethical committee.

\section{Statement of informed consent}

Informed consent was obtained from all individual participants included in the study.

\section{References}

[1] National Institute of Drugs Abuse. Research report series: inhalants. 2012.

[2] Diallo A, Badjabaissi P, Lawson-Evi P, Koffi A, Eklu-Gadegbeku K, Christian M, Edmond C, Messanvi G. Glue abuse in Lome: Investigation and toxicological study on Wister rats. Int J Pharm Pharm Science. 2015; 8(1): 348-352.

[3] Neumark Y, Bar-Hamburger R. Volatile substance misuse among youth in Israel: Results of a national school survey. Subst. Use Misuse. 2011; 46: 21-26.

[4] Verma R, Balhara YPS, Deshpande SN. Inhalant abuse: a study of a tertiary care de-addiction clinic. East Asian Archives Psychiatry. 2011; 21: 157-163.

[5] Elkoussi A, Bakheet S. Volatile substance misuse among street children in Upper Egypt. Subst. Use Misuse. 2011; 46: 35-39.

[6] Garland EL, Howard MO, Vaughn MG. Volatile substance misuses in the United States. Subst. Use Misuse. 2011; 46: 21-26.

[7] Metwally S, Gebrehiwet Y, Yimesghen Y. Glue sniffing and health hazards among street children: an Eritrean field study. Eur. J. Res Soc Science. 2014; 2: 11-28. 
[8] Subramaniam S, Khan HBH, Elumalai N, Lakshmi SYS. Hepatoprotective effect of ethanolic extract of whole plant of Andrographis paniculata against CCl4-induced hepatotoxicity in rats. Comp Clin Pathology. 2015; 24: 1-7.

[9] Howida SAS. Physiological changes due to hepatotoxicity and the protective role of some medicinal plants. Benisuef University Journal of Basic and Applied Sciences. 2016; 48: 1-13.

[10] Otitoju O, Onwurah INE. Biomarkers of Pesticide Contaminated Environment, Pesticides in the Modern World Pests Controland Pesticides Exposure and Toxicity Assessment, Dr. Margarita Stoytcheva (Ed.). 2011.

[11] Reitman S, Frankel S. In-vitro determination of transaminase activity in serum. Am J Clin Pathologz. 1975; 28: 5658.

[12] Singh A, Bhat TK, Sharma OP. Clinical Biochemistry of Hepatotoxicity. J. Clinic Toxicology. 2011; S4: 001.

[13] Jean CD, Donald RH, Qiuhu S, Eugene M, Emmanuel R, Micheal M, Victorien N, Leonard G, Jean DS, Emmanuel M, Kathryn A. Association of abnormal liver function parameters with HIV serostatus and CD4 count in antiretroviral-naïve Rwanda women. Aids Research and Human Retroviruses. 2015; 31(7): 723-730.

[14] Canadian Center on Substance Abuse. Youth volatile substance abuse. 2006.

[15] Dorcas OF. Drug abuse among Nigerian adolescent's strategies for counseling. The Journal of International Social Research. 2012; 5(20): 341-347.

[16] Otmane B, Zakaria O, Saada BM, Nora K, Mohamed B. Acute and chronic glue sniffing effects and consequences of withdrawal on aggressive behavior. Elsevier. 2016; 152: 14-20. 